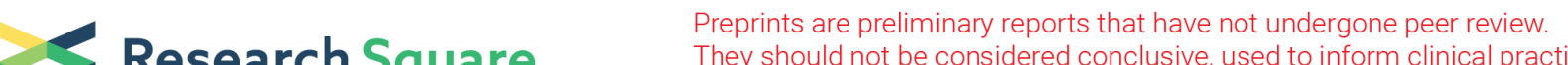 $\begin{array}{ll}\text { Research Square } & \text { They should not be considered conclusive, used to inform clinical practice, } \\ \text { or referenced by the media as validated information. }\end{array}$
}

\section{Mechanism of Hypoxia-Activated MiR-194 /FoxO3a Inducing Glycolytic Metabolism Reprogramming in Gastric Precancerous Lesions}

\section{Sheng-xiong Zhang}

Guangdong Work Injury Rehabilitation Hospital: Guangdong Provincial Work Injury Rehabilitation Center Wei Liu ( $\square$ gzyliuwei@126.com )

Guangzhou University of Chinese Medicine https://orcid.org/0000-0003-3738-5218

\section{Bo Ai}

Tongji Medical College of Huazhong University of Science and Technology: Huazhong University of Science and Technology Tongji Medical College

\section{Hua-feng Pan}

Guangzhou University of Chinese Medicine

\section{Zi-ming Zhao}

Guangzhou University of Chinese Medicine

\section{Zhe-Sheng Chen}

St John's University

\section{Li-zhu Lin}

Guangzhou University of Chinese Medicine

\section{Research Article}

Keywords: Gastric Precancerous Lesion, MNU, Metabolic reprogramming, miR-194-5p, FoxO3a

Posted Date: September 7th, 2021

DOI: https://doi.org/10.21203/rs.3.rs-788905/v1

License: (c) (1) This work is licensed under a Creative Commons Attribution 4.0 International License. Read Full License 


\section{Abstract}

Background: Early diagnosis and treatment of gastric precancerous lesions (GPL) are key factors for reducing the incidence and morbidity of gastric cancer. The study aimed to examine GPL in mice induced by MNU and to illustrate the underlying mechanisms of tumorigenesis.

Methods: In this study, we utilized an in vivo MNU (N-methyl-N-nitroso-urea)-induced GPL mouse model, Histopathological changes of the gastric mucosa were observed by Hematoxylin and Eosin (H\&E-stain) and alcian blue (AB-PAS-stain); The level of miR-194-5p in the gastric mucosa were determined by realtime polymerase chain reaction; We used transmission electron microscopy to observe the effects of MNU on gastric chief cells and parietal cells; We performed immunohistochemical detection of HIF-1a, vWF, Ki-67 and P53, while the changes in protein expression of key genes in LKB1-AMPK and AKT-Fox03 signaling pathways were detected by western blot analysis.

Results: We demonstrated that miR-194-5p expression was upregulated under hypoxia in GPL gastric tissues, and that a high miR-194-5p expression level closely related with tumorigenesis. Mechanistically, miR-194-5p exerted the acceleration of activities related to metabolic reprogramming through LKB1AMPK and AKT-FoxO3 pathways. Furthermore, similar to miR-194-5p, high expression levels of AMPK and AKT were also related to the metabolic reprogramming of GPL. Moreover, we revealed the correlation between the expression levels of miR-194-5p, p-AMPKa, p-AKT, and FoxO3a.

Conclusions: These findings suggest that miR-194-5p/FoxO3 pathway is important for the reversal of metabolic reprogramming in GPL. Thus, exploring strategies to regulate the miR-194-5p/FoxO3a pathway may provide an efficient strategy for the prevention and treatment of GPL.

\section{Introduction}

Gastric cancer is the third leading cause of cancer mortality worldwide in men, and the fifth leading cause of cancer mortality in women(Mattiuzzi and Lippi 2020). Intestinal-type gastric cancer is preceded by $\mathrm{GPL}$, including chronic atrophic gastritis (CAG), intestinal metaplasia (IM), and dysplasia (Dys), finally progressing to cancer(Cahill et al. 1996). Among patients who undergo gastroscopy with biopsy for clinical indications, $2 \%$ of patients with CAG, $2.56 \%$ of patients with IM, and $5.26 \%$ patients with Dys will develop cancer within 20 years(Song et al. 2015). Little is known about the mechanisms of gastric cancer tumorigenesis and development, and GPL could be an important subject for studying the pathogenesis of gastric cancer.

MicroRNAs bind to target genes and subsequently regulate gene expression; as a result, they play a key role in a variety of cell developmental processes and in tumorigenesis. Previous studies have identified differentially expressed miRNAs during early gastric cancer and have demonstrated the specific expression of miR-194-5p in the gastrointestinal tract, which was induced during the process of intestinal epithelial cell differentiation(Hino et al. 2008; Hwang et al. 2018). miR-194-5p plays an important role in 
the invasion and progression of gastric cancer(Song et al. 2012). However, the molecular mechanisms of miR-194-5p in the carcinogenesis of GPL remain to be elucidated.

Metabolism provides information about the progression, and treatment of cancer. Under a hypoxic microenvironment, the Forkhead transcription factors of class 03 (FoxO3) signaling pathway is involved in the regulation of tumor glucose metabolism reprogramming, malignant biological behavior of cancer cells (proliferative autophagy, apoptosis, inflammatory response, etc.), and in the staging, metastasis and prognosis of tumors. Additionally, FoxO3 exhibits tumor suppressive effects on GC, which might be a promising therapeutic target in clinic(Park et al. 2015). FoxO3a, a target of AKT and AMPK pathways, is involved in gastric cancer(Altomare and Testa 2005).

By analyzing differentially expressed miRNAs in GPL compared to normal gastric tissues, we demonstrated that the expression of miR-194-5p is upregulated in GPL. miR-194-5p regulates glucose metabolism of GPL through its targets Fox03a. In addition, we also revealed a positive feedback loop between AMPK and AKT.

\section{Materials And Methods \\ 2.1. Animals}

Adenocarcinomas of the mouse stomach were induced as previously reported(Tomita et al. 2007). Briefly, age-matched male specific pathogen-free (SPF) C57/B6 mice were randomly divided into the control group $(n=15)$ and the model group $(n=15)$. The mice were provided with free drinking MNU(N-methyl-Nnitrosourea) solution (120 ppm) (Aladdin industrial corporation, Shanghai, China) in light-shielded bottles, and were subjected to feed every other day; meanwhile, the control mice were fed normally for 8 weeks. The study protocols were approved by the Ethics Committee of Guangzhou University of Chinese Medicine (No. S2017089). The mice were sacrificed and the stomach was harvested for further experiments.

\subsection{Histology, Transmission Electron Microscopy, and Immunohistochemistry}

Tissue specimens were embedded in paraffin and stained with Hematoxylin and Eosin (H\&E-stain) and alcian blue (AB-PAS-stain) for mucus characterization. For electron microscopy, the samples were placed in $2 \%$ paraformaldehyde and $2.5 \%$ glutaraldehyde at $4^{\circ} \mathrm{C}$, then stained with $1 \%$ uranyl acetate and lead citrate before being observed under the transmission electron microscope. Immunohistochemistry was performed as described by Wei et al.(Liu et al. 2019a)with antibodies to HIF-1a (Millipore, MAB5382), vWF (Abcam, ab194405), Ki-67 (Abcam, ab16667), and P53 (Millipore, CBL404).

\subsection{Western blot}

The following antibodies were used: rabbit FoxO3 antibody (AF6020), rabbit anti-Phospho-FoxO3 antibody (ab154786), Rabbit anti-AMPK alpha antibody (ab133448), rabbit anti-Phospho-AMPK alpha 
antibody (AF3423), Rabbit anti-Phospho-Akt antibody (CST 4060), Rabbit anti-AKT antibody(ab179463), anti-Phospho-LKB1 antibody (AF3453), rabbit anti-PCK1 antibody (DF6770), rabbit anti-GAPDH antibody (AF7021).

\subsection{Real-time PCR}

We adopted the method of $2^{-} \triangle \Delta C T$ to calculate the relative expression levels of miR-194-5p in the gastric mucosa. miR-194-5p was normalized using U6 RNA levels. ${ }^{\triangle} \mathrm{CT}=\mathrm{CT}$ miR-194-5p - CT U6RNA. The primers for the qRT-PCR were as follows: U6:5'-CGCTTCGGCAGCACATATACTA-3' and 5'-

CGCTTCACGAATTTGCGTGTCA-3'; miR-194-5p:5'-

CTAGTACCTAGAGGAACCTTTGAAGACTGTTACAGCTCAGCA - 3' and 5'-: AGCTTGCTGAGCTGTAACAGTCTTCAAAGGTTCCTCTAGGTA-3'.

\section{5. statistical analysis}

All numerical data are expressed as means \pm SEM. Unpaired t-test was performed to determine the statistical significance of the difference between two independent groups. $P<0.05$ was considered statistically significant.

\section{Results}

\subsection{Pathological changes of gastric mucosa in precancerous lesions}

In order to explore the influence of MNU on histopathological injury, H\&E staining was used to evaluate histopathological alterations. In general, the gastric mucosa of mice in the control group was smooth, moist and reddish. The gland arrangement of gastric mucosa in the model group was more irregular than that in the control group. In the MNU-treated group, the gastric mucosa was pale, with poor elasticity, and the thickness was fairly uniform. Some of the sub-mucosal vascular hyperplasia, erosion or granular changes were seen; the epithelial cells were relatively incomplete with uneven cell size and less irregular gland arrangement, which indicated that the GPL model has been successfully established.

We used the AB-PAS staining to evaluate the degree of IM in gastric tissues(Park and Kim 2015). Neutral mucin in the normal mucosa was stained red, while the gastric mucosa in the control group was unstained. After AB staining, the metagenetic tissue of gastric mucosa in mice induced by MNU showed a positive staining. Intestinal metagenetic cells were located in the gastric lumen and lamina propria, and the upper part showed a weak PAS positive staining, with only a small amount of red dye (Fig. 1).

\subsection{Transmission electron microscope analysis}

Next, we used transmission electron microscopy to observe the effects of MNU on gastric chief cells and parietal cells, and showed that the gastric mucosal gland cavity of the mice in the control group was small and consisted of a single layer of gastric mucosal epithelial cells. The chief cells and parietal cells of control mice were large in size, rich in cytoplasm, and had round or oval nuclei. There were abundant 
endoplasmic reticulum and pepsinogen particles in the main cells. In parietal cells, abundant mitochondria could be seen.

Compared with mice in the control group, the gastric mucosal gland cavity of the model group was significantly expanded; the monolayer became pseudostratified glandular epithelium, with a smaller volume of glandular epithelial cells and with increased electron density. The size of the primary and parietal cells of the mice model group was inconsistent, with less cytoplasm and increased nucleoplasm, and there were a large number of vacuoles of different sizes in the cytoplasm. The chief cell vacuoles were concentrated on the side of the gland cavity and appeared to merge into large vesicles. The remaining main cells formed dysplastic cells with a small volume and a large nucleocytoplasmic ratio.

The small vacuoles in the parietal cells were scattered in the cytoplasm, and the original secretory tubule wall membranous structure could be seen in the vacuoles. The fused vacuoles could form sinuses and dysplastic cells. The dense connection between the main parietal cell and the adjacent cell disappeared and transformed into a spindle-shaped epithelial-like transformation cell, indicating ischemia and hypoxia in the gastric mucosa after MNU treatment (Fig. 2).

\subsection{Upregulated expression of HIF-1a and vwf are related to the ischemic hypoxia microenvironment of GPL}

Hypoxia is a typical feature in tumors. In response to hypoxia, HIF-1a and von Willebrand factor (vWF) were activated in cells. vWF staining was used to show tiny vessels and individual endothelial cells. To explore the influence of MNU on mucosal hypoxia and ischemia, a tissue array was used to examine expression of HIF-1a and VWF by IHC staining. The results revealed that the levels of HIF-1a and vWF of MNU-treated mice were both significantly higher than that of control group $(P<0.01)$ (Fig. 3).

\subsection{Upregulated expression of $\mathrm{Ki}-67$ and $\mathrm{P} 53$ are related to the hypoxia microenvironment of GPL}

Ki-67 is a nuclear protein expressed in proliferating cells. P53 mutations can often be detected in the early lesions of gastric cancer (Shimizu et al. 2014). P53 activates hypoxia signaling as early as gastric premalignancy(Sethi et al. 2019). P53 and Ki-67 have been used as reference indicators for predicting the prognosis of gastric cancer (Al-Moundhri et al. 2005; Tzanakis et al. 2009).We performed immunohistochemical detection of P53 and Ki-67 expression in the stomach. Immunohistochemistry showed that upregulated expression of P53 and $\mathrm{Ki}-67$ are related to the hypoxic microenvironment of GPL compared to the control group $(P<0.05)$ (Fig. 4). Altogether, these results suggest that MNU induces gastric mucosal hypoxia in mice.

\subsection{A novel miRNA-194-5p is overexpressed under hypoxia}

Studies showed that miR-194-5p provides the basis for the diagnosis and prognosis of gastric cancer, and is closely related to glycolysis(Song et al. 2012; Zhao et al. 2018). In order to further disclose 
differentially expressed miRNAs in GPL, qRT-PCR assays were conducted to determine the distinct expression patterns of miR-194-5p in gastric mucosa samples from control mice and mice with GPL. The assays showed that miR-194-5p was significantly increased after MNU treatment (Fig. 5). Thus, we demonstrated in this study that upregulation of miR-194-5p in GPL under hypoxic conditions contributed to glycolysis.

\subsection{Changes in protein expression of key genes in FoxO3a signaling pathway in the gastric mucosa}

To investigate the effects of MNU on mice, we examined the expression levels of key gene proteins that regulate glycolysis. The ratio of P-FoxO3a/FoxO3a was lower, and p-AMPK/AMPK and p-AKT/AKT ratios were higher in the model group compared with control group. The expression levels of proteins PCK1 and LKB1 decreased, and the expression of LDHA in the gastric mucosa of the model-mice group increased, compared with the control group (Fig. 6).

\section{Discussion}

The incidence of gastric cancer is very high and it has become one of the leading causes of cancerrelated deaths worldwide. Changes in GPL are linked to an increased risk of gastric cancer(Choi et al. 2016). The reprogramming of cell metabolism is an important sign of cancer and is closely related to the occurrence of tumors. The characteristics of the tumor cells mainly include excessive activation of anaerobic glycolysis and aerobic respiration(Liu et al. 2019b). Epidemiological studies have shown that elevated blood glucose levels are one of the risk factors for gastric cancer(Yamagata et al. 2005). Therefore, elucidating the factors related to glycolysis is essential for the effective prevention of gastric cancer(Tennant et al. 2010). This study is consistent with our previous report that there is glycolysis in the gastric mucosa of GPL(Liu et al. 2020a). MNU induces gastric adenoma and adenocarcinoma due to inflammatory reactions to the alkylating agent(Leung et al. 2008; Tsukamoto et al. 2007; Yamachika et al. 1998), and has been utilized to induce gastric cancer in mice(Tomita et al. 2007; Yoshizawa et al. 2009).

In our study, the mouse model showed IM and Dys of gastric mucosa, and microscopic analysis showed that ischemia and hypoxia could be observed in mice receiving MNU. Thus, we proved that the GPL model was successfully established by short-term gavage of MNU in mice.

The formation of tumor is mainly governed by tissue hypoxia, which is a key molecular feature of the tumor microenvironment(Wang et al. 2020). In the response to hypoxia, angiogenesis(Jain 2014), and reprogramming, energy metabolism(Bristow and Hill 2008) is involved. Hypoxia-induced HIF-1a promotes gastric cancer cell proliferation, invasion, and migration both in vitro and in vivo(Semenza 2012; Xia et al. 2020). The key step in the occurrence and progression of cancer is angiogenesis(Hisano and Hla 2019; Jain et al. 2010). vWF, a multimeric plasma glycoprotein, acts as a marker of endothelial dysfunction(Mandorfer et al. 2018; Sadler 2013). More importantly, vWF has been widely used as a biomarker in gastric cancer(Yang et al. 2018, 2015). However, its functional role in GPL is largely 
unknown. Here, we report that in the gastric mucosa of mice treated with $\mathrm{MNU}$, the expression levels of HIF-1a and vWF were significantly increased. These findings demonstrate the causal role of ischemic hypoxia microenvironment-derived VWF in mediating the carcinogenic characteristics of MNU and identify vWF as a new therapeutic target.

In the clinic, HIF-1 a expression was correlated with aberrant P53 accumulation and cell proliferation(Zhong et al. 1999). Ki-67 is a widely used biomarker to estimate the proportion of dividing cells in order to grade tumors(Scholzen and Gerdes 2000). The presence of strong nuclear staining of P53 in the majority of cancer cells is frequently observed(Baas et al. 1994). P53 and Ki-67 immunostaining indicated that the mice treated with MNU showed more positive cells compared to the control mice. Altogether, here we reported that the ischemic hypoxic microenvironment increased the expression levels of HIF-1 $a$ and vWF, which regulated the level of P53 and promoted the expression of Ki67.

Hypoxia-inducible miRNAs are engaged in the metabolic reprogramming process. An increasing amount of microRNAs have been found to be related to the carcinogenesis and prognosis of gastric cancer patients(Ueda et al. 2010; Yasui et al. 2004, 2009). miR-194-5p promotes gastric carcinogenesis(Sousa et al. 2016). Most of the up-regulated miRNAs have been linked to gastric cancer. For this study, though, we focused on correlations of miRNAs with GPL. In the present study, we showed that miR-194-5p expression levels increased in the GPL. We suggest that miR-194-5p upregulation is an early event in the cascade of events that lead to the conversion of GPL to cancer, and that it contributes to the establishment of an GPL expression profile through regulation of hypoxic microenvironment.

Transcription of genes encoding glycolytic enzymes are activated by HIF-1a, including stimulation of glycolysis by upregulation of LDHA, which creates an acidic tumor microenvironment(Ryan et al. 1998; Zhong et al. 1999). PCK1 is a gluconeogenic enzyme that leads to the regulation of glucose production(Troy et al. 2008). In this study, we also observed changes in gluconeogenic enzymes after MNU treatment as the level of LDHA was increased and the levels of PCK1 decreased in the stomach. In mammalian cells, FoxO3 was involved in glucose metabolism(Kops et al. 2002; Medema et al. 2000). The regulation of FoxO3a by PI3K-AKT and LKB1-AMPK may play a crucial role in controlling energy balance. The PI3K-AKT pathway controls cell survival, proliferation and tumor growth, whereas the LKB1-AMPK pathway controls cell cycle arrest and tumor suppression, and promotes longevity(Hawley et al. 2005). FoxO3a, one of the intersections between both the pathways(Biggs 3rd et al. 1999; Brunet et al. 1999), is inhibited by the PI3K-AKT pathway and activated by the LKB1-AMPK pathway (Greer et al. 2007; Shin et al. 2016). Thus, the PI3K-AKT and the LKB1-AMPK pathways may orchestrate a series of transcriptional (via FoxO3) and post-transcriptional (via mTOR) changes that allow the organism to adapt to changes in the hypoxic status. Our group has characterized the protein expression profiles of PI3K, AKT and mTOR in GPL mice(Liu et al. 2020a, b, a). In the current study, the LKB1 protein expression levels were downregulated, while the ratio of p-AMPK/AMPK and p-AKT /AKT were higher in MNU- receiving mice compared the control mice. Here, we generated the FoxO3a profile of GPL compared to normal mice. We showed evidence here that hypoxia activated miR-194/FoxO3a, inducing glycolytic metabolism 
reprogramming by upregulating the expression of LDHA and downregulating the expression of PCK1 in GPL (Fig. 7).

In summary, our findings identified a novel miR-194-5p, which was up-regulated in GPL. Mechanistically, miR-194-5p functioned as an oncogenic miRNA by regulation of the hypoxic microenvironment and subsequently regulated the PI3K-AKT and the LKB1-AMPK pathways via FoxO3a, inducing reprogramming of glycolytic metabolism.

\section{Declarations}

\section{Conflict of interest}

All authors declare that this project was conducted in the absence of any commercial or financial relationships that could be construed as a potential conflict of interest.

\section{Ethics statement}

The animal study (Protocol \#S2017089) was reviewed and approved by the Ethics Committee of Guangzhou University of Chinese Medicine.

\section{Author contributions}

Wei Liu and Sheng-xiong Zhang designed the study. Sheng-xiong Zhang, Bo Ai, Hua-feng Pan and Ziming Zhao conducted the experiments. Bo Ai and Zi-ming Zhao provided helpful advice for the manuscript. Li-zhu Lin and Zhe-Sheng Chen supervised and revised the manuscript. All authors meet the ICMJE criteria for authorship.

\section{Acknowledgments}

This work was supported by the Natural Science Foundation of China for the Youth (Grant No. 82004300), China Postdoctoral Science Foundation (Grant No. 2019 M662877), the Guangdong Province Basic and Applied Basic Research Fund Project (Grant No.2019A1515110236) and the Natural Science Foundation of China (Grant No. 81973816).

\section{Conflict of interest}

The authors declare that they have no conflicts of interest.

\section{Data Availability Statements}

Authors can confrm that all relevant data are included in the article . 


\section{References}

1. Al-Moundhri MS, Nirmala V, Al-Hadabi I, Al-Mawaly K, Burney I, Al-Nabhani M et al (2005) The prognostic significance of p53, p27 kip1, p21 waf1, HER-2/neu, and Ki67 proteins expression in gastric cancer: a clinicopathological and immunohistochemical study of 121 Arab patients. J Surg Oncol 91(4):243-252. https://doi.org/10.1002/jso.20324

2. Altomare DA, Testa JR (2005) Perturbations of the AKT signaling pathway in human cancer. Oncogene 24(50):7455-7464. https://doi.org/10.1038/sj.onc.1209085

3. Baas IO, Mulder JW, Offerhaus GJ, Vogelstein B, Hamilton SR (1994) An evaluation of six antibodies for immunohistochemistry of mutant p53 gene product in archival colorectal neoplasms. J Pathol 172(1):5-12. https://doi.org/10.1002/path.1711720104

4. Biggs WH 3rd, Meisenhelder J, Hunter T, Cavenee WK, Arden KC (1999) Protein kinase B/Aktmediated phosphorylation promotes nuclear exclusion of the winged helix transcription factor FKHR1. Proc Natl Acad Sci U S A 96(13):7421-7426. https://doi.org/10.1073/pnas.96.13.7421

5. Bristow RG, Hill RP (2008) Hypoxia and metabolism. Hypoxia, DNA repair and genetic instability. Nat Rev Cancer 8(3):180-192. https://doi.org/10.1038/nrc2344

6. Brunet A, Bonni A, Zigmond MJ, Lin MZ, Juo P, Hu LS et al (1999) Akt promotes cell survival by phosphorylating and inhibiting a Forkhead transcription factor. Cell 96(6):857-868. https://doi.org/10.1016/s0092-8674(00)80595-4

7. Cahill RJ, Kilgallen C, Beattie S, Hamilton H, O'Morain C (1996) Gastric epithelial cell kinetics in the progression from normal mucosa to gastric carcinoma. Gut 38(2):177-181. https://doi.org/10.1136/gut.38.2.177

8. Choi E, Hendley AM, Bailey JM, Leach SD, Goldenring JR (2016) Expression of Activated Ras in Gastric Chief Cells of Mice Leads to the Full Spectrum of Metaplastic Lineage Transitions. Gastroenterology 150(4):918-930.e13. https://doi.org/10.1053/j.gastro.2015.11.049

9. Greer EL, Oskoui PR, Banko MR, Maniar JM, Gygi MP, Gygi SP et al (2007) The energy sensor AMPactivated protein kinase directly regulates the mammalian FOXO3 transcription factor. J Biol Chem 282(41):30107-30119. https://doi.org/10.1074/jbc.M705325200

10. Hawley SA, Pan DA, Mustard KJ, Ross L, Bain J, Edelman AM et al (2005) Calmodulin-dependent protein kinase kinase-beta is an alternative upstream kinase for AMP-activated protein kinase. Cell Metab 2(1):9-19. https://doi.org/10.1016/j.cmet.2005.05.009

11. Hino K, Tsuchiya K, Fukao T, Kiga K, Okamoto R, Kanai T et al (2008) Inducible expression of microRNA-194 is regulated by HNF-1alpha during intestinal epithelial cell differentiation. RNA 14(7):1433-1442. https://doi.org/10.1261/rna.810208

12. Hisano Y, Hla T (2019) Bioactive lysolipids in cancer and angiogenesis. Pharmacol Ther 193:91-98. https://doi.org/10.1016/j.pharmthera.2018.07.006

13. Hwang J, Min BH, Jang J, Kang SY, Bae H, Jang SS et al (2018) MicroRNA Expression Profiles in Gastric Carcinogenesis. Sci Rep 8(1):14393. https://doi.org/10.1038/s41598-018-32782-8 
14. Jain RK (2014) Antiangiogenesis strategies revisited: from starving tumors to alleviating hypoxia. Cancer Cell 26(5):605-622. https://doi.org/10.1016/j.ccell.2014.10.006

15. Jain S, Harris J, Ware J (2010) Platelets: linking hemostasis and cancer. Arterioscler Thromb Vasc Biol 30(12):2362-2367. https://doi.org/10.1161/ATVBAHA.110.207514

16. Kops GJ, Dansen TB, Polderman PE, Saarloos I, Wirtz KW, Coffer PJ et al (2002) Forkhead transcription factor FOXO3a protects quiescent cells from oxidative stress. Nature 419(6904):316321. https://doi.org/10.1038/nature01036

17. Leung WK, Wu KC, Wong CY, Cheng AS, Ching AK, Chan AW et al (2008) Transgenic cyclooxygenase2 expression and high salt enhanced susceptibility to chemical-induced gastric cancer development in mice. Carcinogenesis 29(8):1648-1654. https://doi.org/10.1093/carcin/bgn156

18. Liu W, Pan HF, Yang LJ, Zhao ZM, Yuan DS, Liu YL et al (2020a) Panax ginseng C.A. Meyer (Rg3) Ameliorates Gastric Precancerous Lesions in Atp4a-/- Mice via Inhibition of Glycolysis through PI3K/AKT/miRNA-21 Pathway. Evid Based Complement Alternat Med 2020):2672648. https://doi.org/10.1155/2020/2672648

19. Liu W, Yang LJ, Liu YL, Yuan DS, Zhao ZM, Wang Q et al (2020b) Dynamic characterization of intestinal metaplasia in the gastric corpus mucosa of Atp4a-deficient mice. Biosci Rep. https://doi.org/10.1042/BSR20181881

20. Liu W, Zhao ZM, Liu YL, Pan HF, Lin LZ (2019a) Weipiling ameliorates gastric precancerous lesions in Atp4a-/- mice. BMC Complement Altern Med 19(1):318. https://doi.org/10.1186/s12906-019-2718-y

21. Liu Y, Zhang Z, Wang J, Chen C, Tang X, Zhu J et al (2019b) Metabolic reprogramming results in abnormal glycolysis in gastric cancer: a review. Onco Targets Ther 12):1195-1204. https://doi.org/10.2147/OTT.S189687

22. Mandorfer M, Schwabl P, Paternostro R, Pomej K, Bauer D, Thaler J et al (2018) Von Willebrand factor indicates bacterial translocation, inflammation, and procoagulant imbalance and predicts complications independently of portal hypertension severity. Aliment Pharmacol Ther 47(7):980988. https://doi.org/10.1111/apt.14522

23. Mattiuzzi C, Lippi G (2020) Cancer statistics: a comparison between World Health Organization (WHO) and Global Burden of Disease (GBD). Eur J Public Health 30(5):1026-1027. https://doi.org/10.1093/eurpub/ckz216

24. Medema RH, Kops GJ, Bos JL, Burgering BM (2000) AFX-like Forkhead transcription factors mediate cell-cycle regulation by Ras and PKB through p27kip1. Nature 404(6779):782-787. https://doi.org/10.1038/35008115

25. Park SH, Jang KY, Kim MJ, Yoon S, Jo Y, Kwon SM et al (2015) Tumor suppressive effect of PARP1 and FOXO3A in gastric cancers and its clinical implications. Oncotarget 6(42):44819-44831. https://doi.org/10.18632/oncotarget.6264

26. Park YH, Kim N (2015) Review of atrophic gastritis and intestinal metaplasia as a premalignant lesion of gastric cancer. J Cancer Prev 20(1):25-40. https://doi.org/10.15430/JCP.2015.20.1.25 
27. Ryan HE, Lo J, Johnson RS (1998) HIF-1 alpha is required for solid tumor formation and embryonic vascularization. EMBO J 17(11):3005-3015. https://doi.org/10.1093/emboj/17.11.3005

28. Sadler JE (2013) von Willebrand factor in its native environment. Blood 121(14):2583-2584. https://doi.org/10.1182/blood-2013-02-482000

29. Scholzen T, Gerdes J (2000) The Ki-67 protein: from the known and the unknown. J Cell Physiol 182(3):311-322. https://doi.org/10.1002/(SICI)1097-4652(200003)182:3<311::AID-JCP1>3.0.C0;2-9

30. Semenza GL (2012) Hypoxia-inducible factors in physiology and medicine. Cell 148(3):399-408. https://doi.org/10.1016/j.cell.2012.01.021

31. Sethi N, Kikuchi O, McFarland J, Zhang Y, Chung M, Kafker N et al (2019) Mutant p53 induces a hypoxia transcriptional program in gastric and esophageal adenocarcinoma. JCl Insight 4:15. https://doi.org/10.1172/jci.insight.128439

32. Shimizu T, Marusawa H, Matsumoto Y, Inuzuka T, Ikeda A, Fujii Y et al (2014) Accumulation of somatic mutations in TP53 in gastric epithelium with Helicobacter pylori infection. Gastroenterology 147(2):407-417.e3. https://doi.org/10.1053/j.gastro.2014.04.036

33. Shin HJ, Kim H, Oh S, Lee JG, Kee M, Ko HJ et al (2016) AMPK-SKP2-CARM1 signalling cascade in transcriptional regulation of autophagy. Nature 534(7608):553-557. https://doi.org/10.1038/nature18014

34. Song H, Ekheden IG, Zheng Z, Ericsson J, Nyrén O, Ye W (2015) Incidence of gastric cancer among patients with gastric precancerous lesions: observational cohort study in a low risk Western population. BMJ 351:h3867. https://doi.org/10.1136/bmj.h3867

35. Song Y, Zhao F, Wang Z, Liu Z, Chiang Y, Xu Y et al (2012) Inverse association between miR-194 expression and tumor invasion in gastric cancer. Ann Surg Oncol 19(Suppl 3):S509-S517. https://doi.org/10.1245/s10434-011-1999-2

36. Sousa JF, Nam KT, Petersen CP, Lee HJ, Yang HK, Kim WH et al (2016) miR-30-HNF4Y and miR-194NR2F2 regulatory networks contribute to the upregulation of metaplasia markers in the stomach. Gut 65(6):914-924. https://doi.org/10.1136/gutjnl-2014-308759

37. Tennant DA, Durán RV, Gottlieb E (2010) Targeting metabolic transformation for cancer therapy. Nat Rev Cancer 10(4):267-277. https://doi.org/10.1038/nrc2817

38. Tomita H, Yamada Y, Oyama T, Hata K, Hirose Y, Hara A et al (2007) Development of gastric tumors in $\mathrm{Apc}(\mathrm{Min} /+)$ mice by the activation of the beta-catenin/Tcf signaling pathway. Cancer Res 67(9):4079-4087. https://doi.org/10.1158/0008-5472.CAN-06-4025

39. Troy S, Soty M, Ribeiro L, Laval L, Migrenne S, Fioramonti X et al (2008) Intestinal gluconeogenesis is a key factor for early metabolic changes after gastric bypass but not after gastric lap-band in mice. Cell Metab 8(3):201-211. https://doi.org/10.1016/j.cmet.2008.08.008

40. Tsukamoto T, Mizoshita T, Tatematsu M (2007) Animal models of stomach carcinogenesis. Toxicol Pathol 35(5):636-648. https://doi.org/10.1080/01926230701420632

41. Tzanakis NE, Peros G, Karakitsos P, Giannopoulos GA, Efstathiou SP, Rallis G et al (2009) Prognostic significance of p53 and Ki67 proteins expression in Greek gastric cancer patients. Acta Chir Belg 
109(5):606-611. https://doi.org/10.1080/00015458.2009.11680496

42. Ueda T, Volinia S, Okumura H, Shimizu M, Taccioli C, Rossi S et al (2010) Relation between microRNA expression and progression and prognosis of gastric cancer: a microRNA expression analysis. Lancet Oncol 11(2):136-146. https://doi.org/10.1016/S1470-2045(09)70343-2

43. Wang X, Che X, Yu Y, Cheng Y, Bai M, Yang Z et al (2020) Hypoxia-autophagy axis induces VEGFA by peritoneal mesothelial cells to promote gastric cancer peritoneal metastasis through an integrin a5fibronectin pathway. J Exp Clin Cancer Res 39(1):221. https://doi.org/10.1186/s13046-020-01703-x

44. Xia X, Wang S, Ni B, Xing S, Cao H, Zhang Z et al (2020) Hypoxic gastric cancer-derived exosomes promote progression and metastasis via MiR-301a-3p/PHD3/HIF-1a positive feedback loop. Oncogene 39(39):6231-6244. https://doi.org/10.1038/s41388-020-01425-6

45. Yamachika T, Nakanishi H, Inada K, Tsukamoto T, Shimizu N, Kobayashi K et al (1998) N-methyl-Nnitrosourea concentration-dependent, rather than total intake-dependent, induction of adenocarcinomas in the glandular stomach of BALB/c mice. Jpn J Cancer Res 89(4):385-391. https://doi.org/10.1111/j.1349-7006.1998.tb00575.x

46. Yamagata H, Kiyohara Y, Nakamura S, Kubo M, Tanizaki Y, Matsumoto T et al (2005) Impact of fasting plasma glucose levels on gastric cancer incidence in a general Japanese population: the Hisayama study. Diabetes Care 28(4):789-794. https://doi.org/10.2337/diacare.28.4.789

47. Yang AJ, Wang M, Wang Y, Cai W, Li Q, Zhao TT et al (2018) Cancer cell-derived von Willebrand factor enhanced metastasis of gastric adenocarcinoma. Oncogenesis 7(1):12. https://doi.org/10.1038/s41389-017-0023-5

48. Yang X, Sun HJ, Li ZR, Zhang H, Yang WJ, Ni B et al (2015) Gastric cancer-associated enhancement of von Willebrand factor is regulated by vascular endothelial growth factor and related to disease severity. BMC Cancer 15:80. https://doi.org/10.1186/s12885-015-1083-6

49. Yasui W, Oue N, Ito R, Kuraoka K, Nakayama H (2004) Search for new biomarkers of gastric cancer through serial analysis of gene expression and its clinical implications. Cancer Sci 95(5):385-392. https://doi.org/10.1111/j.1349-7006.2004.tb03220.x

50. Yasui W, Oue N, Sentani K, Sakamoto N, Motoshita J (2009) Transcriptome dissection of gastric cancer: identification of novel diagnostic and therapeutic targets from pathology specimens. Pathol Int 59(3):121-136. https://doi.org/10.1111/j.1440-1827.2009.02329.x

51. Yoshizawa N, Yamaguchi H, Yamamoto M, Shimizu N, Furihata C, Tatematsu M et al (2009) Gastric carcinogenesis by N-Methyl-N-nitrosourea is enhanced in $\mathrm{db} / \mathrm{db}$ diabetic mice. Cancer Sci 100(7):1180-1185. https://doi.org/10.1111/j.1349-7006.2009.01157.x

52. Zhao X, Hou Y, Tuo Z, Wei F (2018) Application values of miR-194 and miR-29 in the diagnosis and prognosis of gastric cancer. Exp Ther Med 15(5):4179-4184.

https://doi.org/10.3892/etm.2018.5931

53. Zhong H, De Marzo AM, Laughner E, Lim M, Hilton DA, Zagzag D et al (1999) Overexpression of hypoxia-inducible factor 1 alpha in common human cancers and their metastases. Cancer Res 59(22):5830-5835 


\section{Figures}

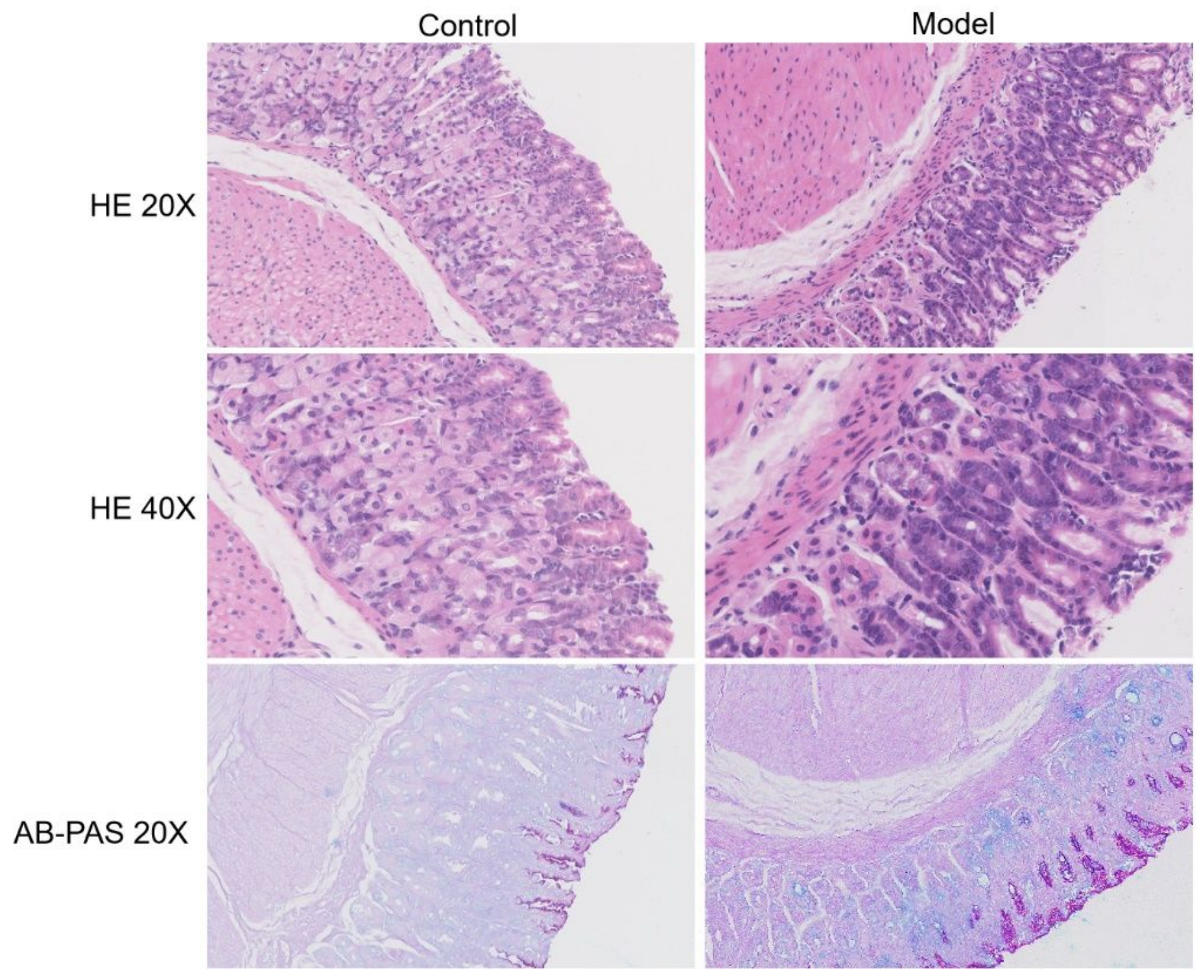

Figure 1

Effect of MNU on gastric mucosal damage in mice. H\&E staining (top panels); and AB-PAS staining (bottom panel). 
Control

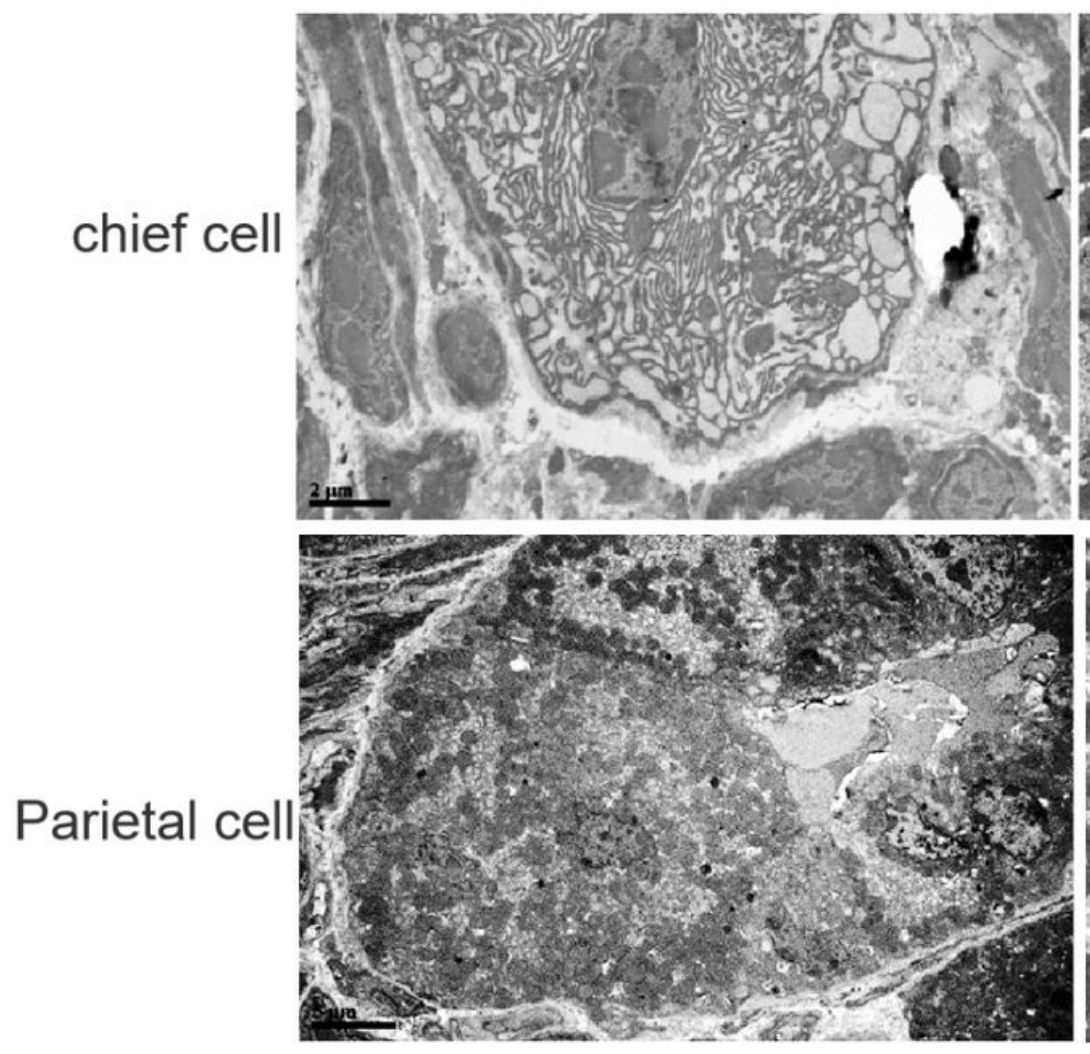

Model
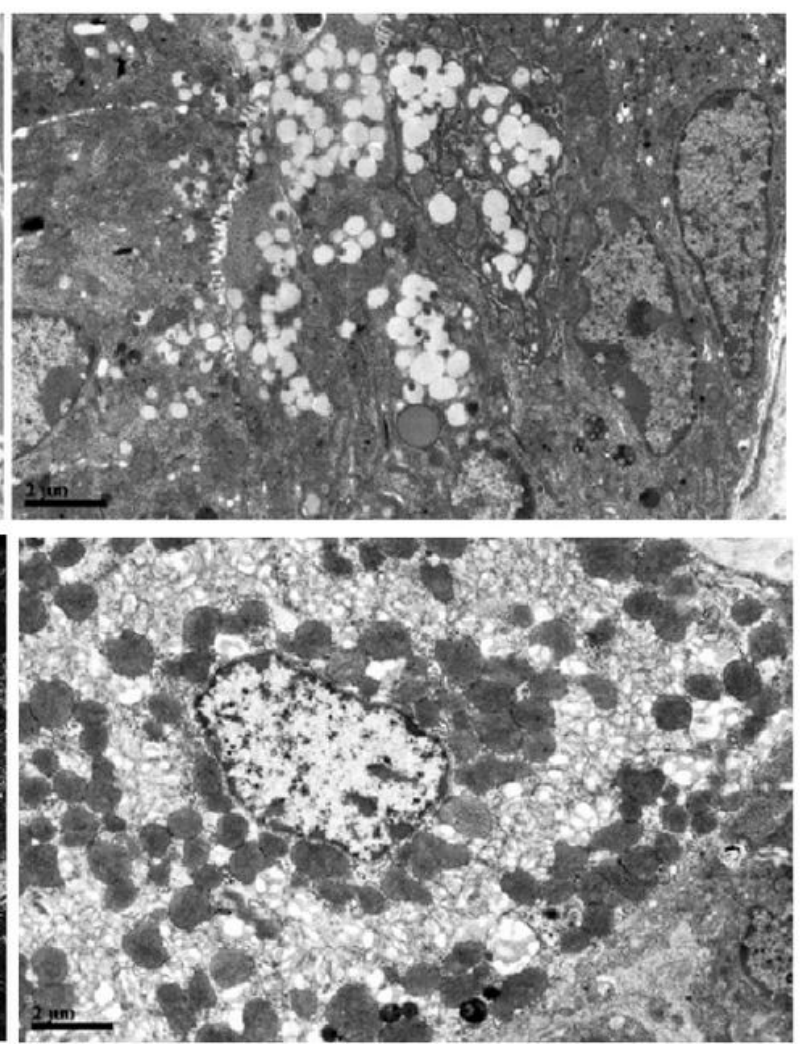

\section{Figure 2}

MNU induces ischemia and hypoxia in gastric mucosa. Ultrastructural changes of chief cells (top panels) and parietal cells (bottom panels) in gastric mucosa. 

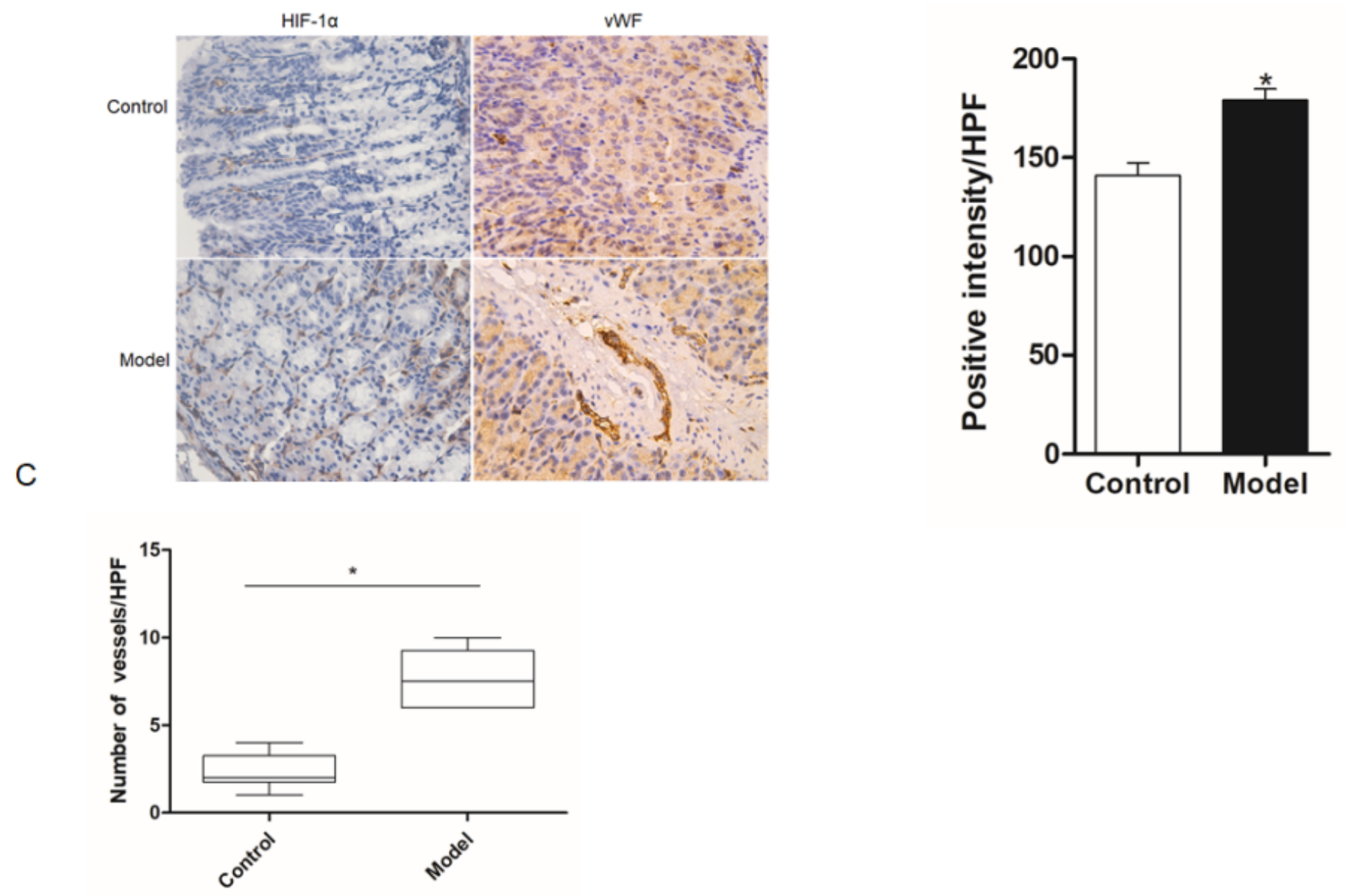

Figure 3

MNU induces a hypoxia transcriptional program in GPL. A, A tissue array was used to examine expression of HIF-1 $a$ and vWF by IHC. The Boxplot diagrams visualizing the number of stained vessels per high power field (HPF), C and B, the statistical analysis of HIF-1 $a$ and vWF $* \mathrm{P}<0.01$ compared with control group.

A

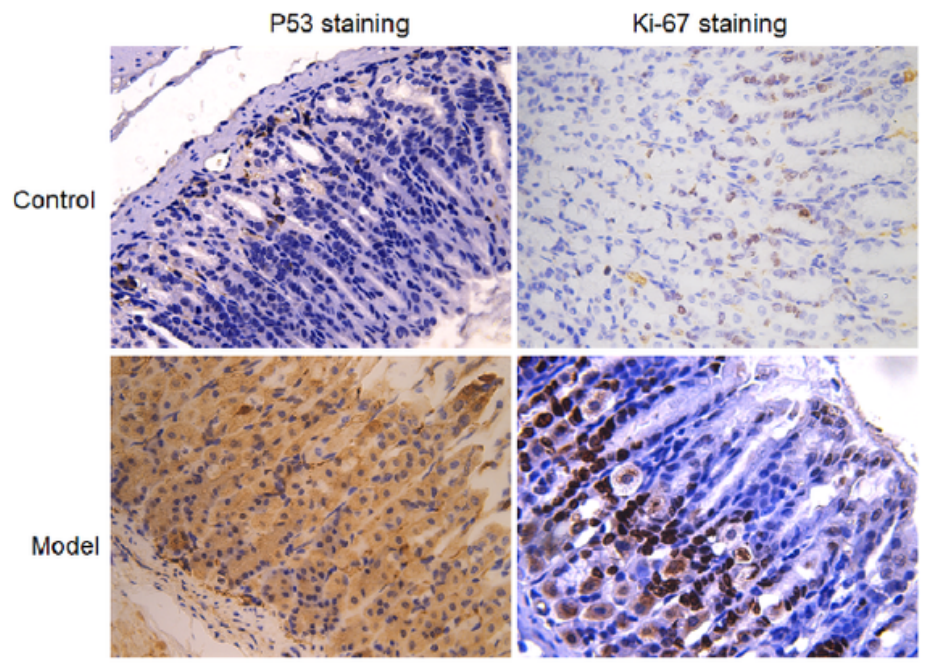

B

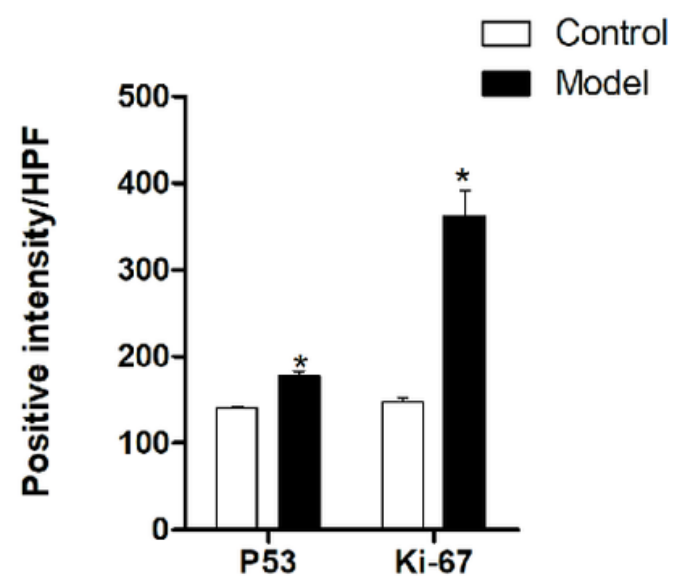


Figure 4

P53 and Ki-67 were increased in GPL. A, IHC staining of P53 and Ki-67. B, The statistical analysis of P53 and Ki-67. ${ }^{\mathrm{P}}<0.05$ compared with control group.

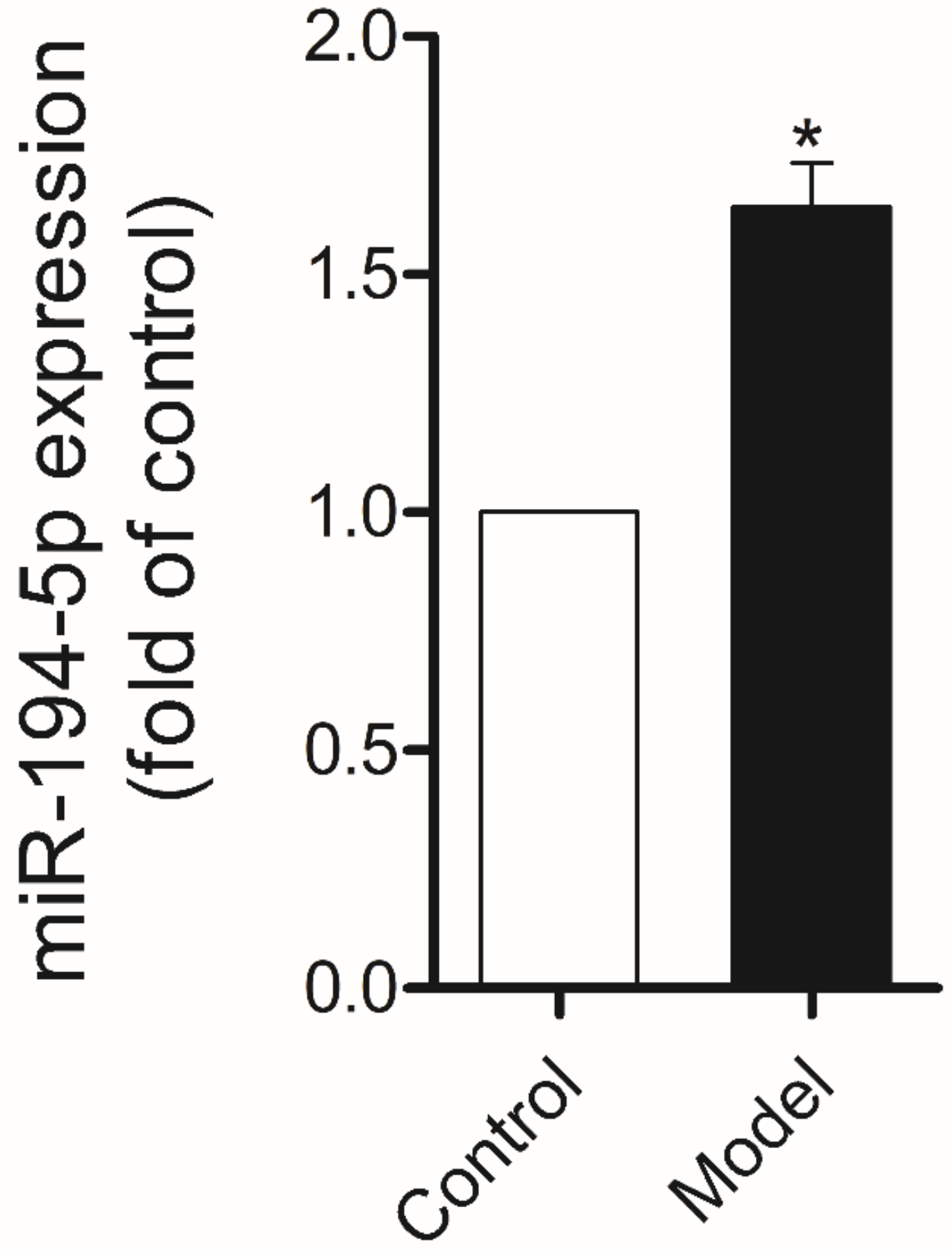

Figure 5 
The expression of miR-194-5p increases in the gastric mucosa of GPL. ${ }^{\mathrm{P}}<0.05$ compared with control group.

A

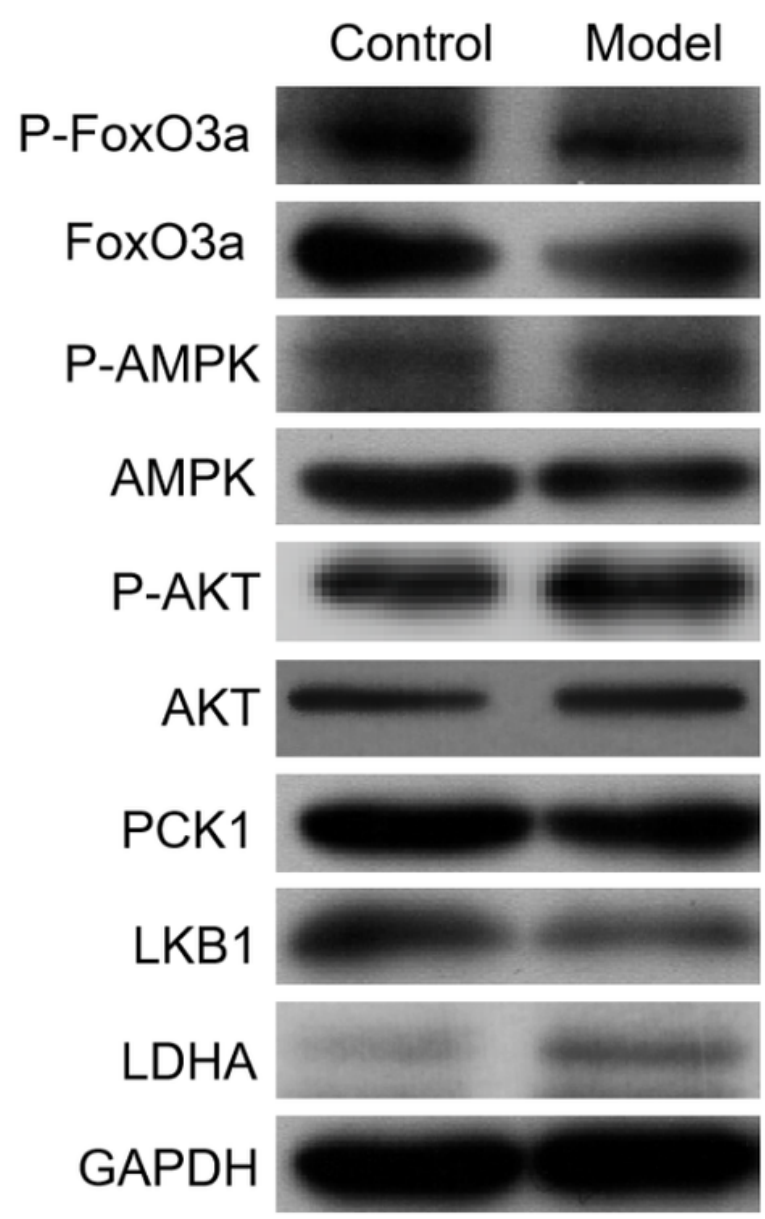

B

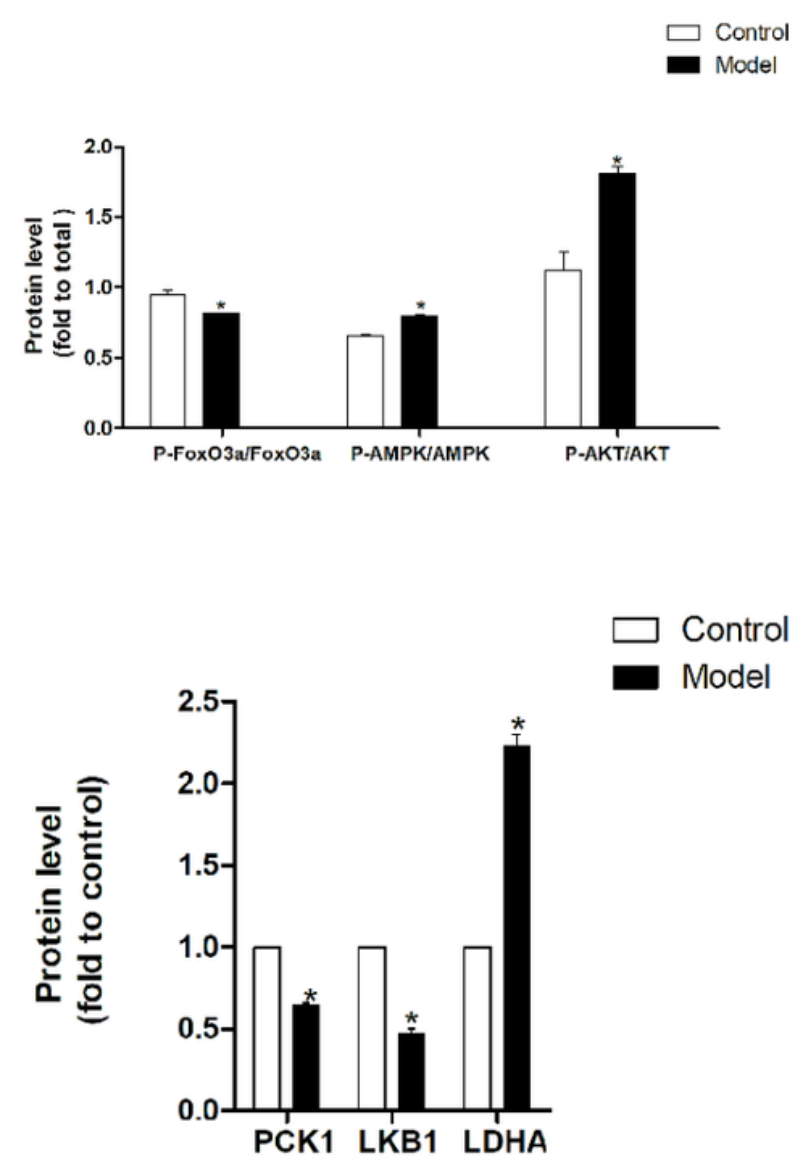

Figure 6

Changes in expression levels of key proteins in the FoxO3a signaling pathway in gastric mucosa. $A$, immunoblot showing protein expression levels of P-FoxO3a, FoxO3a, P-AMPK, AMPK, PCK1, LKB1, and LDHA. B, The ratio of P-Fox03a/FoxO3a is lower, P-AMPK/AMPK and P-AKT/AKT are higher in GPL.C, The PCK1, LKB1 decreased, and LDHA overexpressed in GPL, *Pख0.05. 


\section{miR-194-5p}

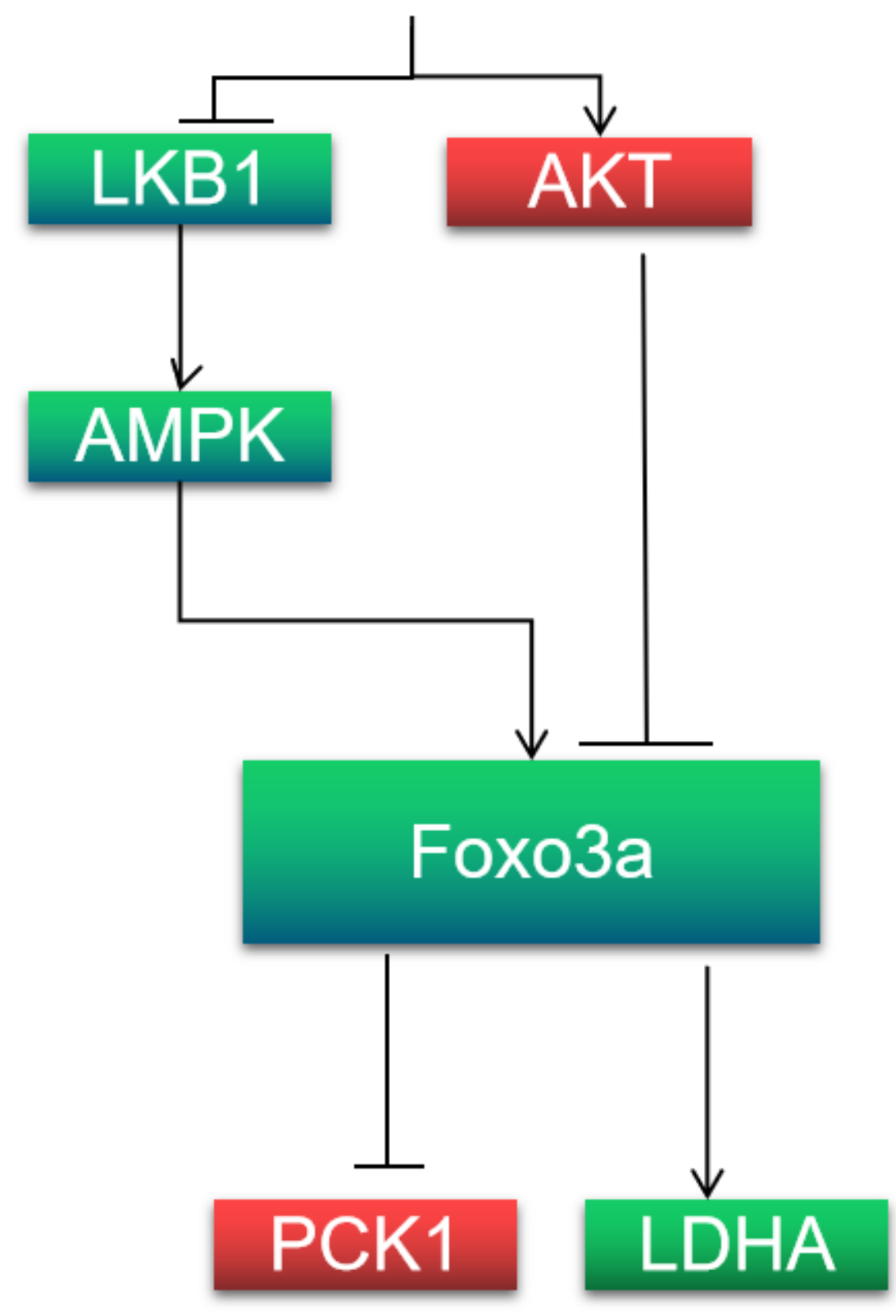

Figure 7

Schematic representation of the mechanism by which miR-194-5p exerted the acceleration of activities related to metabolic reprogramming. miR-194-5p protects against hypoxia in GPL gastric tissues by regulation of the hypoxic microenvironment and subsequently regulated the PI3K-AKT and the LKB1AMPK pathways via Fox03a.

\section{Supplementary Files}

This is a list of supplementary files associated with this preprint. Click to download. 
- supplementary.doc

Page 19/19 